\title{
AVALIAÇÃO ELETROMIOGRÁFICA DO MÚSCULO MASSETER EM PESSOAS COM PARALISIA FACIAL PERIFÉRICA DE LONGA DURAÇÃO
}

\author{
Masseter muscle electromyographic assessment \\ in subject with long lasting facial palsy
}

\author{
Adriana Rahal ${ }^{(1)}$, Maria Valéria Schmidt Goffi-Gomez ${ }^{(2)}$
}

\begin{abstract}
RESUMO
Objetivo: verificar a atividade elétrica do músculo masseter em pessoas com paralisia facial periférica de longa duração. Métodos: participaram deste estudo seis sujeitos de ambos os sexos, com paralisia facial há pelo menos doze meses, sem queixas mastigatórias e sem disfunção temporomandibular e com pelo menos seis dentes em cada hemiarcada. Todos preencheram um questionário de anamnese e em seguida foram submetidos à eletromiografia de superfície dos masseteres de ambos os lados. As provas eletromiográficas foram: posição habitual com lábios fechados, apertamento dentário, mastigação habitual e unilateral à direita e à esquerda com uva passa. Resultados: em todas as provas eletromiográficas não foram observadas diferenças significantes $(p=0,05)$ entre os lados com e sem paralisia facial. Conclusão: observou-se com o presente estudo que a força do músculo masseter não sofre influência da paralisia facial de longa duração.
\end{abstract}

DESCRITORES: Paralisia Facial; Músculo Masseter; Mastigação; Eletromiografia

\section{INTRODUÇÃO}

A paralisia facial pode influenciar a vida da pessoa em diversos aspectos pessoais como nas funções da vida diária, na comunicação e nos aspectos emocionais ${ }^{1}$. Muitos tratamentos incluindo exercícios, estimulação elétrica, biofeedback já foram descritos como meios de tratamento para paralisia facial com resultado bastante positivo ${ }^{2}$. A combinação entre a mímica e fisioterapia possibilita um rápido avanço na musculatura orofacial e minimiza os problemas com a alimentação, fala e integração social ${ }^{3}$. Diversos são os meios utilizados na terapia miofuncional que têm como objetivo a melhora muscular e funcional do indivíduo acometido pela paralisia facial periférica ${ }^{4}$, entretanto, a literatura pesquisada, não descreve o que ocorre com indivíduos com paralisia facial periférica de longa duração que provavelmente apresentam preferência mastigatória pelo lado contralateral à pa-

(1) Fonoaudióloga; CEFAC - Saúde e Educação; Doutoranda pelo Departamento de Otorrinolaringologia da Faculdade de Medicina da Universidade de São Paulo.

(2) Fonoaudióloga; Hospital das Clínicas da Faculdade de Medicina da Universidade de São Paulo; Doutora em Ciências pela Universidade Federal de São Paulo. ralisia em função da pouca mobilidade muscular do lado paralisado.

Em uma mastigação bilateral, o alimento é distribuído de forma homogênea, favorecendo a pressão uniforme das forças mastigatórias sobre os tecidos de suporte do dente. Deste modo, a atividade dos músculos mastigatórios (temporal, masseter, pterigóideos, bucinador e supra-hióideos) quando bilateral e sincrônica, facilita a estabilidade dos tecidos periodontais e da oclusão ${ }^{5}$.

$\mathrm{Na}$ mastigação unilateral, a musculatura mastigatória, especialmente dos músculos masseter, temporal e bucinador, apresenta maior força muscular no lado do trabalho, lado onde à mastigação está ocorrendo. A musculatura do lado de balanceio, lado contrário ao lado do trabalho, encontra-se, via de regra, mais alongada e com tônus funcional diminuído, podendo até haver uma assimetria muscular visualmente perceptível ${ }^{6}$.

Indivíduos com paralisia facial periférica potencialmente têm condições que induzem à mastigação unilateral, realizada pelo lado não afetado, principalmente pela dificuldade de ação do músculo bucinador (inervado pelo nervo facial) uma vez que com a pouca participação deste músculo, há acúmulo de resíduos do lado afetado. 
Dentre os músculos elevadores, o masseter tem grande importância nos ciclos mastigatórios por ser um músculo de muita potência e um dos grandes responsáveis pela trituração do alimento ${ }^{7}$. Desta maneira, pode-se supor que o indivíduo com paralisia facial periférica flácida de longa duração venha a apresentar alteração nos músculos masseteres em função de uma mastigação unilateral pós-paralisia de longa duração.

O presente estudo teve como objetivo verificar a atividade elétrica do músculo masseter em pessoas com paralisia facial periférica de longa duração.

\section{MÉTODOS}

O grupo constituiu-se de seis sujeitos de ambos os sexos, com paralisia facial há pelo menos doze meses sem cirurgias reparadoras na face, sem queixas mastigatórias, sem disfunção da articulação temporomandibular e com pelo menos seis dentes em cada hemiarcada. Após consentimento livre e esclarecido e o preenchimento do questionário de anamnese, todos foram submetidos ao exame eletromiográfico de superfície. Utilizou-se o eletromiógrafo modelo K6-I Light Channel Surface Eletromyography (Myo-Tronics Co. Seattle, WA), de oito canais, com scanner \#9, calibrado a uma amplitude de 500 microvolts (mV). Os eletrodos superficiais duplos de superfície de Cloreto de Prata descartáveis (Duo-Trodes, Myo-tronics, Seattle, WA) foram aderidos bilateralmente sobre os músculos masseteres e o eletrodo terra sobre o músculo esternocleidomastóideo.

A gordura da pele foi removida com gaze embebida em álcool $70^{\circ}$. As provas eletromiográficas foram:

1. posição habitual com lábios unidos com duração de 15 segundos.

2. apertamento dentário: o indivíduo foi orientado a apertar os dentes durante 3 segundos e três momentos distintos.

3. mastigação habitual: o indivíduo foi orientado a mastigar três uvas passas do modo em que está habituado.
4. mastigação à direita: o indivíduo foi orientado a mastigar três uvas passas do lado direito.

5. mastigação à esquerda: o indivíduo foi orientado a mastigar três uvas passas do lado esquerdo.

Todos os sujeitos foram avaliados pela mesma examinadora e todos os exames foram realizados sempre no período entre 8:00h e 10:00h da manhã.

Este trabalho foi apresentado e aprovado pelo comitê de Ética do Hospital das Clínicas da Faculdade de Medicina da USP (HCFMUSP) sob protocolo $n^{\circ}$ 169/06.

As respostas eletromiográficas de cada prova de ambos os lados da face expressas em microvolts $(\mu \mathrm{V})$ resultantes da média final bruta da atividade elétrica em uma janela de 15 segundos foram submetidas à análise estatística realizada com o teste $\mathrm{t}$ student, considerando-se $p=0,05$.

\section{RESULTADOS}

Os dados dos indivíduos estudados estão demonstrados na Tabela 1. A idade média da população estudada, dois do sexo masculino e quatro do feminino, foi de 47 anos, com tempo médio de instalação da PFP de 33 meses.

$\mathrm{Na}$ Tabela 2, está demonstrada a atividade eletromiográfica, durante posição habitual. A média eletromiográfica total do lado com PFP foi de $2,5 \mu \mathrm{V}$ e do lado sem PFP foi de 2,9 $\mu \mathrm{V}$. Na Tabela 3, verifica-se a prova de apertamento dentário, onde a média eletromiográfica total do lado com PFP foi de 93,6 $\mu \mathrm{V}$ e sem PFP de 76,8 $\mu \mathrm{V}$. Na Tabela 4, está demonstrada a média da prova de mastigação habitual, com média eletromiográfica total do lado com PFP de 19,5 $\mu \mathrm{V}$ e sem PFP de 19,4 $\mu \mathrm{V}$. Nas Tabelas 5 e 6, foram computadas as provas de mastigação unilateral à direita e à esquerda e as médias eletromiográficas totais foram, na Tabela 5 de $13,4 \mu \mathrm{V}$ lado com PFP e 26,0 $\mu \mathrm{V}$ lado sem PFP e na Tabela 6, 19,4 $\mu$ V lado com PFP e 22, $1 \mu$ V lado sem PFP.

Em todas as provas eletromiográficas não foram observadas diferenças significantes $(p=0,05)$ entre os lados com e sem paralisia facial (PFP).

Tabela 1 - Dados demográficos dos pacientes avaliados

\begin{tabular}{ccccc}
\hline & $\begin{array}{c}\text { Média etária } \\
\text { (em anos) }\end{array}$ & sexo & $\begin{array}{c}\text { Tempo médio de } \\
\text { instalação daPFP } \\
\text { (em meses) }\end{array}$ & Etiologia \\
\hline 1 & 56 & F & 18 & Bell \\
2 & 30 & M & 30 & Traumática \\
3 & 34 & F & 50 & Bell \\
4 & 48 & F & 17 & Traumática \\
5 & 60 & F & 25 & Traumática \\
6 & 54 & M & 58 & Traumática \\
\hline & PFP: paralisia facial periférica & F: feminino & M: masculino
\end{tabular}


Tabela 2 - Amplitude da atividade eletromiográfica (em $\mu \mathrm{V}$ ) à prova posição habitual (PH) com lábios unidos

\begin{tabular}{lcc}
\hline & EMG PH lado PFP & EMG PH lado s/ PFP \\
\hline Caso 1 & 1,6 & 2,2 \\
Caso 2 & 2,2 & 2,7 \\
Caso 3 & 2,9 & 6,9 \\
Caso 4 & 2,3 & 1,7 \\
Caso 5 & 3,0 & 2,2 \\
Caso 6 & 2,9 & 1,5 \\
Média total & 2,5 & 2,9 \\
Desvio padrão & 0,5 & 2,9 \\
& & $\mathrm{p}=0,663$ \\
\hline
\end{tabular}

EMG: eletromiografia de superfície

PH: posição habitual

PFP: paralisia facial periférica

$\mu \mathrm{V}$ : microvolts

Tabela 3 - Amplitude da atividade eletromiográfica (em $\mu \mathrm{V}$ ) à prova de apertamento dentário (AD)

\begin{tabular}{lcc}
\hline & EMG AD lado PFP & EMG AD lado s/ PFP \\
\hline Caso 1 & 70,5 & 42,0 \\
Caso 2 & 38,6 & 31,3 \\
Caso 3 & 40,6 & 55,9 \\
Caso 4 & 85,0 & 68,0 \\
Caso 5 & 123,7 & 56,6 \\
Caso 6 & 203,4 & 206,9 \\
Média total & 93,6 & 76,8 \\
Desvio padrão & 62,3 & 65,0 \\
& & $\mathrm{p}=0,656$ \\
\hline
\end{tabular}

EMG: eletromiografia de superfície

AD: apertamento dentário

PFP: paralisia facial periférica

$\mu \mathrm{V}$ : microvolts

Tabela 4 - Amplitude da atividade eletromiográfica (em $\mu \mathrm{V}$ ) à prova de mastigação habitual (MH)

\begin{tabular}{lcc} 
& EMG MH lado PFP & EMG MH lado s/ PFP \\
\hline Caso 1 & 4,1 & 9,4 \\
Caso 2 & 8,7 & 13,6 \\
Caso 3 & 12,5 & 31,4 \\
Caso 4 & 22,2 & 22,8 \\
Caso 5 & 40,7 & 18,7 \\
Caso 6 & 28,8 & 20,2 \\
Média total & 19,5 & 19,4 \\
Desvio padrão & 13,8 & 7,6 \\
& & $\mathrm{p}=0,982$ \\
\hline
\end{tabular}

EMG: eletromiografia de superfície

$\mathrm{MH}$ : mastigação habitual

PFP: paralisia facial periférica

$\mu \mathrm{V}$ : microvolts 
Tabela 5 - Amplitude da atividade eletromiográfica $(\mathrm{em} \mu \mathrm{V})$ à prova de mastigação unilateral à direita (MD)

\begin{tabular}{|c|c|c|c|}
\hline & Lado da PFP & $\begin{array}{l}\text { EMG MD } \\
\text { lado PFP }\end{array}$ & $\begin{array}{c}\text { EMG MD lado } \\
\text { s/ PFP }\end{array}$ \\
\hline Caso 1 & & 14,9 & 13,4 \\
\hline Caso 2 & & 9,0 & 13,8 \\
\hline Caso 3 & & 8,8 & 27,0 \\
\hline Caso 4 & & 24,2 & 21,9 \\
\hline Caso 5 & & 13,1 & 30,1 \\
\hline Caso 6 & & 10,5 & 49,6 \\
\hline Média total & & 13,4 & 26,0 \\
\hline \multirow[t]{2}{*}{ Desvio padrão } & & 5,8 & 13,4 \\
\hline & & & $p=0,062$ \\
\hline
\end{tabular}

EMG: eletromiografia de superfície

MD: mastigação à direita

PFP: paralisia facial periférica

$\mu \mathrm{V}$ : microvolts

Tabela 6 - Amplitude da atividade eletromiográfica $(\mathrm{em} \mu \mathrm{V})$ à prova de mastigação unilateral à esquerda (ME)

\begin{tabular}{|c|c|c|c|}
\hline & Lado da PFP & $\begin{array}{c}\text { EMG ME lado } \\
\text { PFP }\end{array}$ & $\begin{array}{l}\text { EMG ME lado } \\
\text { s/ PFP }\end{array}$ \\
\hline Caso 1 & & 13,1 & 15,9 \\
\hline Caso 2 & & 8,2 & 17,3 \\
\hline Caso 3 & & 15,7 & 23,6 \\
\hline Caso 4 & & 16,2 & 22,5 \\
\hline Caso 5 & & 7,7 & 43,3 \\
\hline Caso 6 & & 55,4 & 10,0 \\
\hline Média total & & 19,4 & 22,1 \\
\hline \multirow[t]{2}{*}{ Desvio padrão } & & 18,0 & 11,5 \\
\hline & & & $p=0,762$ \\
\hline
\end{tabular}

EMG: eletromiografia de superfície

ME: mastigação à esquerda

PFP: paralisia facial periférica

$\mu \mathrm{V}$ : microvolts

\section{DISCUSSÃO}

Na prática em Motricidade Orofacial, quando existe uma queixa específica, os exames subjetivos podem levar à dificuldade de constatação de relações causa - efeito, tornando os diagnósticos pouco precisos. Os avanços da tecnologia, expandindo o conhecimento, trouxeram novas técnicas tanto de diagnóstico quanto de terapêutica miofuncional, direcionando de maneira objetiva o trabalho fonoaudiológico ${ }^{8}$.

A avaliação eletromiográfica permite o estudo da atividade muscular, possibilitando a captação dos potenciais de ação gerados durante a contração dos músculos, os quais podem ser analisados considerando-se os parâmetros duração e amplitude. A duração (geralmente mensurada em milisegundos) refere-se ao tempo em que determinado grupo muscular permaneceu contraído ou relaxado, enquanto a amplitude (em microvolts) representa o recrutamento das unidades motoras para a execução do movimento, podendo estar relacionada à força muscular ${ }^{9}$.

A colocação dos eletrodos deve seguir a direção perpendicular às fibras musculares. Os eletrodos devem ser fixados na região onde o músculo tem maior volume e maior massa possibilitando a captação da resposta do maior número de unidades motoras ${ }^{10}$. 
A utilização da eletromiografia na Fonoaudiologia é recente e tem sido bastante explorada na área de Motricidade Orofacial. Realizou-se estudo da ação da musculatura supra-hióidea durante a deglutição, em indivíduos com oclusão dentária normal e alterada, chegando à conclusão que esta musculatura possui maior atividade elétrica em indivíduos com oclusão dentária alterada ${ }^{11}$. Trabalhos que analisaram a ação dos masseteres em indivíduos com oclusão normal chegaram à conclusão que existe um equilíbrio de atividade elétrica deste músculo durante a mastigação ${ }^{12}$. A atividade elétrica dos masseteres varia de acordo com a consistência do alimento ${ }^{13}$.

Estudos eletromiográficos dos músculos masseteres realizados em indivíduos com oclusão dentária normal, indicam que o uso dos dentes como "ferramentas de trabalho" e a utilização de alimentos mais fibrosos e duros são fundamentais para aumento de força mastigatória e conseqüente aumento dos valores eletromiográficos ${ }^{14,15}$.

Em indivíduos com paralisia facial periférica que por diversas razões permanecem por longos perìodos na fase flácida, a falta de função muscular prolongada em uma hemiface poderia acarretar diferenças significativas na dinâmica da alimentação considerando-se as limitações impostas pela flacidez do bucinador e da incompetência labial do mesmo lado ${ }^{16}$. Isto pode ocorrer porque do lado da paralisia o músculo bucinador, que é um dos responsáveis pela manutenção do alimento sob a fase oclusal, durante o processo de trituração e pulverização do alimento, acaba não agindo e pode vir a causar alguma assimetria muscular e facial, como reportado por autora ${ }^{16}$.

De fato, seria esperada uma diferença, entre os lados, na mastigação considerando-se a dificuldade do indivíduo mastigar do lado da paralisia facial pela fraqueza do músculo bucinador e do orbicular da boca deste lado, sobretudo pela manutenção prolongada do padrão. Entretanto, na literatura pesquisada, não foram encontrados estudos que referem se há dife- rença de atividade elétrica entre os masseteres em indivíduos com paralisia facial periférica.

Neste estudo, revelou-se que os masseteres não sofreram influência do lado paralisado uma vez que não se encontrou relação entre o lado preferencial da mastigação com o lado acometido pela paralisia facial periférica. Os fatores interferentes no lado preferencial da mastigação são os tipos de oclusão dentária, o número de dentes em cada hemiarcada e as alterações nas articulações temporo mandibulares como referido por autor ${ }^{17}$, uma vez que a trituração e pulverização dos alimentos dependem muito da ação dos dentes. Em outro estudo ${ }^{18}$ verificou-se que em pessoas saudáveis com dentadura natural completa, apenas $10 \%$ apresentam mastigação bilateral simultânea, $75 \%$ apresentam padrão mastigatório bilateral alternado e $15 \%$ mastigação unilateral direita ou esquerda.

Pode-se supor então, que o indivíduo com paralisia facial periférica quando não tem fatores interferentes apresenta dois momentos distintos do processo mastigatório. Quando em fase inicial, ainda em adaptação e com a musculatura bastante flácida do lado da paralisia, não consegue ou evita mastigar deste lado pelo acúmulo de alimento, por uma deficiência de ação do músculo bucinador. Após alguns meses, deve ocorrer uma adaptação da musculatura orofacial e o indivíduo passa a poder mastigar também do lado acometido pela paralisia, muitas vezes auxiliando com a mão.

Será importante então, um estudo comparativo entre os achados eletromiográficos e a avaliação clínica miofuncional que poderá trazer dados significativos auxiliando ainda mais nesta questão.

\section{CONCLUSÃO}

Os dados obtidos, neste estudo, levam a concluir que a força do músculo masseter não sofre interferência da paralisia facial periférica flácida de longa duração.

\section{ABSTRACT}

Purpose: to check the masseter electrical activity in long lasting facial paralysis patients. Methods: six subjects, with facial paralysis for over a period of twelve months, males and females, took part in this study. Patients should not show any masticatory complaints or have any diagnoses of temporomandibular joint dysfunction, having at least six teeth in each half dental ridge. All subjects filled out a questionnaire regarding oral habits and were assessed by surface electromyography of the masseter muscle of both sides. Electromyographic records were taken with lips closed at rest, teeth tightness, besides usual mastication, and unilateral mastication on both sides with raisins. Results: in all electromyographic tests there were no statistically significant differences $(p=0.05)$ between both sides, with and without facial paralysis. Conclusion: it was observed that the strength of the masseter muscle is not under the influence of long lasting facial paralysis.

KEYWORDS: Facial Paralysis; Masseter Muscle; Mastication; Electromyography 


\section{REFERÊNCIAS}

1. Diels HJ. Facial paralysis: is there a role for a therapist? Facial Plast Surg. 2000;16 (4): 361-4.

2. Novak CB. Rehabilitation strategies for facial nerve Injuries. Semin Plast Surg. 2004; 18 (1): 47-51.

3. Beurskens CHG, Heymans PG. Positive effects of mime therapy on sequel of facial paralysis: stiffness, lip mobility, and social and physical aspects of facial disability. Otol Neurotol. 2003; 24: 677-81.

4. Diels HJ, Combs D. Neuromuscular retraining for facial paralysis. Otolaryngol Clin North Am. 1997; 30(5): 727-43.

5. Jabur LB. Avaliação fonoaudiológica. In: Ferreira FV. Ortodontia - diagnóstico e planejamento clínico. 4. ed. São Paulo: Artes Médicas; 2001. p.275-301.

6. Douglas CR. Fisiologia do ato mastigatório. In: Patofisiologia oral: fisiologia normal e patológica aplicada e odontologia e fonoaudiologia. São Paulo: Pancast; 1998. p. 245-72.

7. Berretin-Felix G, Genaro KF, Trindade IEK, Trindade Junior AS. Masticatory function in temporomandibular dysfunction patients: electromyographic evaluation. J Apl Oral Science. 2005;13(4): 360-5.

8. Saito I, Saito Y. Biofeedback training in clinical settings. Biogenic Amines. 2004;18(3):463-76.

9. Bianchini EMG. Mastigação e ATM: avaliação e terapia. In: Marchesan IQ. Fundamentos em fonoaudiologia: aspectos clínicos da motricidade oral. Rio de Janeiro: Guanabara-Koogan. 1998. p.37-49. 10. Ferrario VF, Sforza C, Miani A Jr, D'Addona A, Barbini E. Electromyographic activity of human masticatory muscles in normal young people: statistical evaluation of reference values for clinical applications. J Oral Rehabil. 1993;20:271-80.

11. Rahal A, Lopasso FP. Eletromiografia dos músculos masseteres e supra-hióideos em mulheres com oclusão normal e com má-oclusão classe I de Angle durante a fase oral da deglutição. Rev CEFAC. 2004;6(4):370-5.

12. Rodrigues KA, Ferreira LP. Masseter muscles electromyography study of individuals with and without malocclusion during dental clenching. Electromyogr Clin Neurophysiol. 2004; 44(5): 271-5. 13. Muñoz GC, Silva C, Misaki JK, Rahal A. Análise dos potenciais elétricos do músculo masseter durante a mastigação de alimentos com rigidez variada. Rev CEFAC. 2004; 6(2): 127-34.

14. Jardini RSR. Avaliação facial a partir da relação eletromiográfica e antropométrica do músculo bucinador. Rev Soc Bras Fonoaudiol. 2005; 10(3): 161-8.

15. Falda V, Guimarães A, Bérzin F. Eletromiografia dos músculos masseteres e temporais durante a deglutição e mastigação. Rev APCD 1998; 52(2): 151-7.

16. Goffi-Gomez MVS, Vasconcelos LGE, Bernardes DFF. Intervenção fonoaudiológica na paralisia facial. In: Ferreira LP, Befi-Lopes DM, Limongi SCO, organizadores. Tratado de fonoaudiologia. São Paulo: Roca; 2004.p.512-26.

17. Cattoni DM. Alterações da mastigação. In: Ferreira LP, Befi-Lopes DM, Limongi SCO, organizadores. Tratado de fonoaudiologia. São Paulo: Roca; 2004.p.222-91.

18. Posselt U. Phisiology of occlusion and rehabilitation. Oxford: Blackwell, 1968.
RECEBIDO EM: 17/02/2007

ACEITO EM: 31/05/2007

Endereço para correspondência:

Rua Dr. José Rodrigues Alves Sobrinho, $879^{\circ}$ andar

São Paulo - SP

CEP: 05466-040

Fax: (11) 38680818

E-mail: adrianarahal@cefac.br 


\title{
FONOAUDIOLOGIA E ESTÉTICA FACIAL: ESTUDO DE CASOS
}

\section{Speech therapy and facial esthetic: cases studies}

\author{
Carolina Paes ${ }^{(1)}$, Paula Nunes Toledo ${ }^{(2)}$, Hilton Justino da Silva ${ }^{(3)}$
}

\begin{abstract}
RESUMO
Objetivo: caracterizar as modificações presentes no comportamento facial após tratamento fonoaudiológico proposto para face, através da comparação das medidas da projeção do sulco nasogeniano ao tragus pré e pós-tratamento fonoaudiológico Métodos: a população foi constituída de 10 professores atendidos no Hospital dos Servidores do Estado de Pernambuco de ambos os sexos, e com idade entre 33 e 63 anos. $O$ processo de coleta de dados consistiu de protocolos de anamnese e avaliação, protocolo de desempenho, além do registro fotográfico. Foi realizada a medida da projeção do sulco nasogeniano ao tragus. Durante dezesseis sessões foram trabalhados exercícios isotônicos, isométricos, isocinéticos, massagens, manipulações faciais, equilíbrio funcional estomatognático, além de orientações aos cuidados faciais. Resultados: foi constatada uma redução das medidas da projeção do sulco nasogeniano ao tragus em ambos os lados, observando-se também um notório equilíbrio entre os lados direito e esquerdo. Conclusão: o programa de tratamento proposto para face reduziu as medidas da projeção do sulco nasogeniano ao tragus e proporcionou um equilíbrio entre essas medidas.
\end{abstract}

DESCRITORES: Fonoaudiologia; Face; Estética

\section{INTRODUÇÃO}

O fato de o fonoaudiólogo dominar os conhecimentos específicos das funções vitais como respirar, sugar, mastigar, deglutir e falar permite que o trabaIho na área da Fonoaudiologia estética transcorra de forma harmônica e equilibrada ${ }^{1}$.

É observado um estreito relacionamento entre as marcas e vincos de expressão ao redor da boca e o uso da musculatura oral, visto que rugas indesejá-

(1) Fonoaudióloga do Hospital dos Servidores do Estado de Pernambuco; Coordenadora e professora da Unidade Especializada em Voz e Face (VOCAL \& FACE); Docente do curso de Fonoaudiologia da Faculdade Integrada do Recife; Mestre em Anatomia Patológica pela Universidade Federal de Pernambuco.

(2) Fonoaudióloga; Docente do Centro Universitário das Faculdades Metropolitanas Unidas e do curso de pós-graduação do CEFAC - Saúde Educação; Mestre em Fonoaudiologia pela Pontifícia Universidade Católica de São Paulo; Doutoranda em Ciências, pela Faculdade de Medicina da Universidade de São Paulo, Área de Cirurgia Plástica.

(3) Fonoaudiólogo docente do curso de Fonoaudiologia da Faculdade Integrada do Recife e da Universidade Federal de Pernambuco; Coordenador e professor do Centro de Estudo e Pesquisa em Motricidade Orofacial; Doutor em Nutrição pela Universidade Federal de Pernambuco. veis podem ser resultantes de posturas e movimentos repetidos realizados para mastigar, deglutir, respirar e falar, bem como tais rugas podem sofrer influência da tensão exagerada dos músculos da face ${ }^{2}$.

O relacionamento da motricidade oral influindo nas questões da estética, da beleza e do rejuvenescimento, bem como na suavização das rugas de expressão facial é observado por meio do trabalho integrado entre a Fonoaudiologia e a Dermatologia, evidenciando que este trabalho em conjunto pode prevenir e diminuir a presença dessas rugas, através do reequilíbrio das funções estomatognáticas e do relaxamento da musculatura da face ${ }^{3}$.

A preocupação com os princípios da Fonoaudiologia e estética foi o motivo para a elaboração do Programa de Aprimoramento Muscular em Fonoaudiologia Estética Facial (PAMFEF), onde é preconizada a importância da harmonia anatômica e funcional ${ }^{4}$.

A eficiência dos exercícios isométricos na musculatura da mímica da face também pode ser analisada, quando utilizada em favor da estética visando diminuir a flacidez muscular, rugas e marcas de expressão. Observou-se que, a realização sistemática dos exercícios isométricos pelo período de três meses mostrou favorecer a diminuição das rugas, marcas de expressão e flacidez facial, com variação do grau e localização da melhora ${ }^{5}$. 